\title{
Enhance the chromatic uniformity and luminous efficiency of WLEDs with triple-layer remote phosphor structures
}

\author{
Nguyen Thi Phuong Loan ${ }^{1}$, Anh Tuan Le ${ }^{2}$ \\ ${ }^{1}$ Faculty of Fundamental 2, Posts and Telecommunications Institute of Technology, Vietnam \\ ${ }^{2}$ Faculty of Electrical and Electronics Engineering, Ton Duc Thang University, Vietnam
}

\begin{tabular}{l} 
Article Info \\
\hline Article history: \\
Received Sep 28, 2019 \\
Revised May 18, 2020 \\
Accepted May 27, 2020 \\
\hline Keywords: \\
Color rendering index \\
Dual-layer phosphor \\
Luminous efficacy \\
Mie-scattering theory \\
Remote-phosphor \\
Triple-layer phosphor
\end{tabular}

\begin{abstract}
The angular color uniformity (ACU) with the ability to evaluate chromatic performance of WLED has become an important target to achieve in producing higher-quality WLEDs. This paper studies the ACU enhancing effects of novel triple-phosphor configuration in lighting devices with remote phosphor structure. Moreover, the optical influences of remote phosphor structure with three phosphor layers (TL) on WLEDs properties are calculated and compared to the dual-layer (DL) one for reference. The experiments are applied to devices at 5 distinct correlated color temperature ranging from $5600-8500 \mathrm{~K}$. The results presented that DL structure attains better color rendering index (CRI) than the TL one. Meanwhile, in terms of color quality scales (CQS), TL model shows higher values at all ACCTs, compared to the DL. Moreover, the luminous flux of DL configuration is lower than that of TL structure. In addition, the diversion of color temperature depicts as D-CCT in TL structure is much better than the value in DL structure, especially at high ACCT as $8500 \mathrm{~K}$, which means TL is good for chromatic uniformity of high ACCTs WLEDs. These results proved that the triple-layer structure is superior and more effective to apply for acquiring the enhancement of WLEDs package.
\end{abstract}

Copyright $@ 2020$ Institute of Advanced Engineering and Science. All rights reserved.

\section{Corresponding Author:}

Anh Tuan Le,

Faculty of Electrical and Electronics Engineering,

Ton Duc Thang University,

No. 19 Nguyen Huu Tho Street, Tan Phong Ward, District 7, Ho Chi Minh City, Vietnam.

Email: leanhtuan1@tdtu.edu.vn

\section{INTRODUCTION}

Due to the efficiency in lighting and energy-saving, the diodes with light radiating ability called LEDs has soon recognized as potential lighting method. The applications of LEDs are spread out in many different fields from general usage of street lighting, back-lighting to more advanced utilization in medical and automobile [1-3]. To produce white light, the most common approach is to apply the combination of chromatic lights which yielded from the blue LED chip and the phosphor that ejects light downward. Such configuration is distinguished based on the appearance of phosphor component and known as phosphorconverted LEDs (pc-LEDs). Although the procedure to apply phosphor on the structure and create pc-LEDs are available which consists of the dispersing and conformal methods, these approaches all have drawbacks that are detrimental to pc-LEDs quality. For instance, the luminous efficiency of WLEDs from these methods are usually poor due to the light loss caused by back-scattering lights $[4,5]$. Then, a configuration that separate the phosphor material and blue light source was introduced to enhance light output and known as the remote phosphor (RP) structure. The RP structure creates a gap between the components that helps reduce the re-emission from phosphor, and therefore is able to enhance the optical properties of pc-LEDs [6, 7]. In addition, the RP configuration can reduce the absorption of back-scattered light by the LED chip, thus promoting the reliability of WLEDs and decreasing the junction temperature [8-13]. 
In recent years, the optical properties of RP LEDs have been significantly improved, especially their luminous efficiency. However, their chromatic uniformity is still in low-quality, especially for planar RP configurations [14-16]. In general, a diffuser sheet is a tool to maintain uniformity in allocation and radiation of correlated color temperature (CCT). However, the results show the energy loss in the process of light transmission through the sheet [17-19]. Though resulting in low light efficiency, these approaches get better color uniformity. Besides, although angular color uniformity (ACU) can be adjusted through many different configurations that have been proposed such as the patterned sapphire substrate (PSS) [19], the DBR structure [20], and the dichroic filter that recycles light [21], but high expenses and complexity when implement on an available illuminating network are the disadvantages of these configuration. Therefore, to create a cost-efficient structure that is easy to integrate and has high color uniformity and luminous output, the remote micro-patterned phosphor (RMPP) layer is proposed as the alternative for the standard flat RP film in WLEDs. The RMPP layer is a structure with multiple layers where the flat RP film is laid in the middle of the micro-patterned (MP) polydimethylsiloxane (PDMS) layers. Adapting this idea, dual-layer remote phosphor structures were proposed and investigated to achieve better optical performance. A duallayer RP is comprised of a yellow YAG: $\mathrm{Ce}^{3+}$ layer placed under a red or green phosphor one.

In 2017, Nhan and his partners reported that structure with red phosphor $\mathrm{SrO} \cdot 3 \mathrm{~B}_{2} \mathrm{O}_{3}: \mathrm{Sm}^{2+}$ in the dual remote phosphor can enhance light output by $17 \%$ in comparison to the conventional phosphor configuration. Besides, in the stable concentration of red phosphor $\mathrm{SrO} .3 \mathrm{~B}_{2} \mathrm{O}_{3}: \mathrm{Sm}^{2+}$, the structure can achieve $5 \%$ higher color rendering index (CRI) than the mixed phosphor WLEDs [22, 23]. However, this study just showed the better results of CRI which is one of elements using to evaluate the color quality. Thus, it cannot completely assure the benefit of this structure to color quality of WLEDs, though the luminous flux is better. Another value called color quality scales (CQS) which includes CRI, viewer's preference and color coordinates is consider a more powerful index to evaluate the color quality of WLEDs. Thus, Lee et al. introduced the two packages of dual-layer remote phosphor structures to enhance the lumen output, CRI and CQS of WLEDs. One of them uses $\mathrm{SrBaSiO}_{4}: \mathrm{Eu}^{2+}$ green phosphor layer while the other uses red phosphor $\mathrm{Sr}_{\mathrm{w}} \mathrm{F}_{\mathrm{x}} \mathrm{B}_{\mathrm{y}} \mathrm{O}_{\mathrm{z}}$ : $\mathrm{Eu}^{2+}, \mathrm{Sm}^{2+}$ layer to place above the yellow YAG:Ce ${ }^{3+}$ layer. The results showed that the greenyellow structure can increase the green light component to better the luminous flux but it leads to a large decline in color quality. Meanwhile, the red-yellow model has better rendering ability that expresses through CRI, CQS and resulting in better color quality, although inferior in lumen efficacy. Besides, Anh's team also researched on the different shapes of phosphor layers in the structure which are the flat dual-remote phosphor (FDRP) with planar layer and the concave dual-remote phosphor (CDRP) with indented layer. They finally figured out that compared to CDRP structure, the FDRP can accomplished better luminous flux as the light go through two phosphor layers more easily. They also suggested the suitable distances which are $\mathrm{d}_{1}=0.08$ $\mathrm{mm}$ or $\mathrm{d}_{2}=0.63 \mathrm{~mm}$ to be the gap among the two phosphor layers and the led chip's surface to achieve highest luminous flux. However, they also reported a significant reduction in the color uniformity in both cases. In general, dual-layer remote phosphor can enhance the optical properties of WLEDs, but it hard to attain better luminous flux and color quality at the same time. Therefore, a triple-layer remote phosphor is proposed to improve those aspects in LED production. A triple-layer remote phosphor structure consists of three different phosphor layers: the yellow YAG: $\mathrm{Ce}^{3+}$ is placed at the bottom, covering the nine LED chips, while the red phosphor film is located at the top and the green phosphor layer is the middle. That this structure includes green and red phosphor, not only the green but also the red-light components are increases, leading to the possibility of achieving both higher lumen output and color uniformity simultaneously. Hence, this study will investigate the use of triple-layer remote phosphor to enhance the performance of WLEDs. Moreover, comparisons between the effects of DL structure and TL structure on luminous flux, CRI and CQS will be demonstrated to assured the benefits of the using three phosphor layers inside the LED packages. Thus, we believe that the results from this paper are valuable information for manufacturers to produce WLEDs having better performance.

The phosphor structure in this study is a triple remote phosphor with yellow YAG:Ce ${ }^{3+}$, green $\mathrm{Ca}_{2} \mathrm{La}_{2} \mathrm{BO}_{6.5}: \mathrm{Pb}^{2+}$, and red $\mathrm{Mg}_{8} \mathrm{Ge}_{2} \mathrm{O}_{11} \mathrm{~F}_{2}: \mathrm{Mn}^{4+}$ phosphor layers. The arrangement of studied contents is preparation process of green and red phosphor and the organization of these phosphor layers will be presented in section 2. Section 3 includes the mathematic system used to analyze the performance of the remote phosphor layer, and discuses the attained results. Finally, the conclusion is demonstrated in section 4.

\section{RESEARCH METHOD}

\subsection{Preparation of phosphor materials}

The green phosphor $\mathrm{Ca}_{2} \mathrm{La}_{2} \mathrm{BO}_{6.5}: \mathrm{Pb}^{2+}$ is utilized to increase the internal green light component of WLEDs, and thus enhancing the luminous output and color uniformity. Besides, the red-light component inside WLEDs can be improved by using the red $\mathrm{Mg}_{8} \mathrm{Ge}_{2} \mathrm{O}_{11} \mathrm{~F}_{2}: \mathrm{Mn}^{4+}$ phosphor, leading to higher values of CRI and CQS. The preparations of these two phosphors are demonstrated as follows. 
To prepare $\mathrm{Ca}_{2} \mathrm{La}_{2} \mathrm{BO}_{6.5}: \mathrm{Pb}^{2+}$ phosphor, the steps must be put into a strict order. Initially, the materials are mixed by using dry grinding or milling method. Next, they are fired in open quartz boats with air flows, at about $500^{\circ} \mathrm{C}$. After that, the mixture goes through powderizing process. Then, it is put into capped quartz tubes with $\mathrm{N}_{2}$, and fired for the second time under $1000^{\circ} \mathrm{C}$, in an hour. In the next step, this material will be powderized again. Finally, it will go through the third firing process at $1200^{\circ} \mathrm{C}$ with the presence of $\mathrm{N}_{2}$ flows, for 1 hour.

For the preparation of $\mathrm{Mg}_{8} \mathrm{Ge}_{2} \mathrm{O}_{11} \mathrm{~F}_{2}: \mathrm{Mn}^{4+}$, the process is simpler as it consists of fewer steps than that of $\mathrm{Ca}_{2} \mathrm{La}_{2} \mathrm{BO}_{6.5}: \mathrm{Pb}^{2+}$ phosphor. Firstly, the materials used in this process are mixed by dry ball-milling. Secondly, the mixture is fired with air flows in capped quartz tubes at $1200^{\circ} \mathrm{C}$ for 2 hours. Thirdly, the dry ball-milling method is used to grind the fired substance into powder. Finally, the powder is re-fired in open quartz boats at the same temperature $1200^{\circ} \mathrm{C}$ for approximately 16 hours (overnight).

Before simulating $\mathrm{Ca}_{2} \mathrm{La}_{2} \mathrm{BO}_{6.5}: \mathrm{Pb}^{2+}$ and $\mathrm{Mg}_{8} \mathrm{Ge}_{2} \mathrm{O}_{11} \mathrm{~F}_{2}: \mathrm{Mn}^{4+}$ phosphor, measurements of the materials properties such as concentration, particle size, stimulation spectrum, absorption spectrum, and emission spectrum must be ensured by experiments. However, among the measurements, we need to identify the suitable concentration of phosphor and particle size to achieve the best index in chromatic performance and lighting output. Meanwhile, the parameters of the phosphor spectrum are constants. Based on the results from previous researches, the particles of phosphor have a width of $14.5 \mu \mathrm{m}$.

\subsection{Simulation process}

The cross-sectional schematics in Figure 1 are the illustration of LEDs packages used in the research. Figure 1(a), 1(b), respectively provides visual presentation on the arrangement of WLEDs package with glass cover, phosphor layers, a half-dome, and package with conformal coating. Specifically, the blue areas indicate lighting-emitting chips, the chromatic lines represent the phosphor of the corresponding color, and the empty spaces are the silicone matrix lenses. The substrate for the structure is predetermined as aluminum nitride and the default yellow phosphor is $\mathrm{YAG}: \mathrm{Ce}^{3+}$.

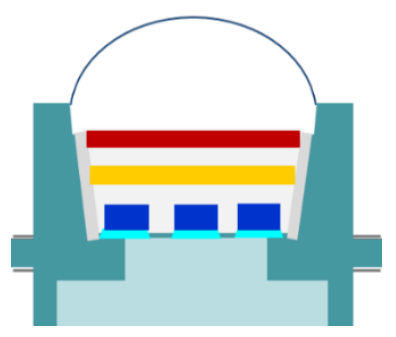

(a)

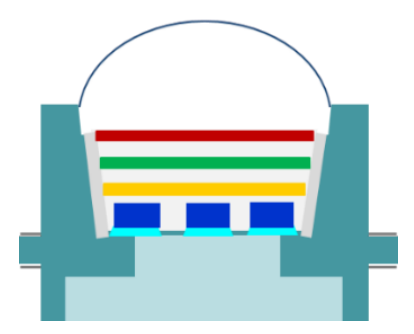

(b)

Figure 1. Illustration of multi-layer phosphor structures of white LEDs, (a) dual-layer phosphor (DL) and (b) triple-layer phosphor (TL)

This density of the phosphors layers is predetermined at $0.08 \mathrm{~mm}$. Besides, the concentration of YAG: $\mathrm{Ce}^{3+}$ changes following the variation of the red or green or red phosphor concentration to maintain the average correlated color temperatures (ACCTs). In addition, at each different ACCT of each phosphor structure, the YAG: $\mathrm{Ce}^{3+}$ concentrations are diverse, which creates the diversity of scattering characteristic inside WLEDs. Moreover, this discrepancy leads to the differences in optical properties.

It can be seen from Figure 2 that at all ACCTs, the yellow YAG:Ce ${ }^{3+}$ phosphor concentration in DL structure higher than in TL structure. Considering the same ACCT in all structures, if the YAG:Ce ${ }^{3+}$ concentration is higher, there will be more back-scattering events, and the reduced emitted luminous flux is higher. On the other hand, when the concentration of YAG: $\mathrm{Ce}^{3+}$ is raised, the imbalance among the three primary colors that produce white lights, including yellow, red and green, will appear, leading to the reduction in color quality. Hence, to advance the luminous flux and the chromatic quality of WLEDs, the back-scattering needs to be reduced by increasing the red-light component. Besides, the chromatic performance as well as the light output also under influences of green light. According to the mentioned information, it seems that the triple-layer phosphor structure is the most advantageous one, in terms of managing optical properties. In order to prove this assumption, the research team continues to provide other essential references to the remote phosphor structures. In Figure 3 is the measured emission spectra in both dual and triple-layer configurations. From the results, the emission spectrum of TL structure is higher than DL structure at several wavelength ranges which suggests that pc-LEDs with three phosphor layers has better lighting efficiency regardless of color performance. 


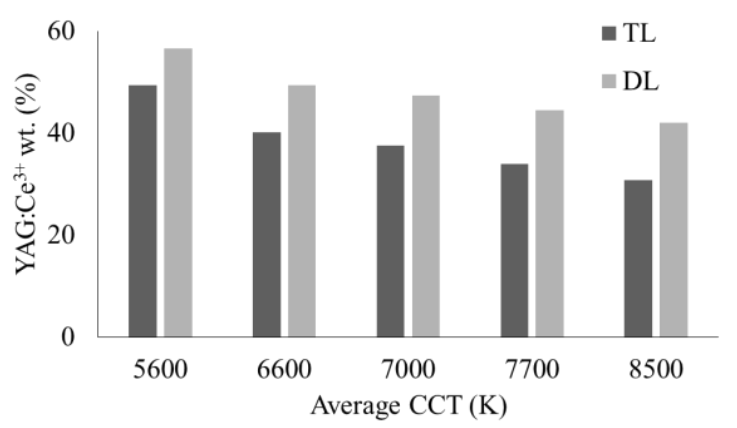

Figure 2. The concentration of yellow YAG: $\mathrm{Ce}^{3+}$ phosphor in each remote phosphor structure at each different ACCT

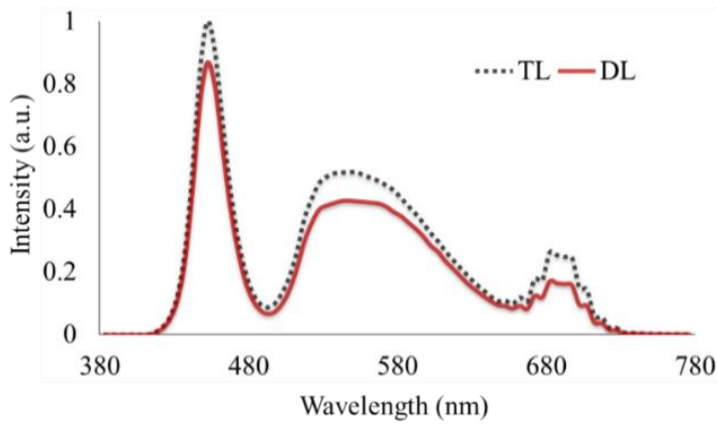

Figure 3. Emission spectra of phosphor configurations

\section{RESULTS AND ANALYSIS}

Figure 4 demonstrates the CRI measured from dual-layer (DL) and triple-layer (TL) remote phosphor configurations that are used to research for phosphor influences. As can be seen, DL structure shows higher CRI than the TL, regardless of ACCT. Moreover, CRI of DL structure rises along with each of ACCT and reaches the highest value at ACCT of $8500 \mathrm{~K}$. This result is one of the most vital references to improve CRI for remote phosphor structures, especially, when controlling the CRI is hard to achieve at high ACCT (over $7000 \mathrm{~K}$ ). This structure is beneficial to the CRI because of the added red-light component from the red $\mathrm{Mg}_{8} \mathrm{Ge}_{2} \mathrm{O}_{11} \mathrm{~F}_{2}: \mathrm{Mn}^{4+}$ phosphor layer. Meanwhile, in terms of CRI, the TL structure is the runner-up. Therefore, it can be assured that, with the objective as CRI, it is possible to choose DL structure in mass-producing WLEDs. However, CRI is just one of the indexes used to evaluate the color quality. In recent years, another parameter called CQS has become popular to many researches. CQS is a combination of the three elements: CRI, viewers' preference, and chromaticity coordinate. Due to its cover of these three factors, CQS has been a great goal of researchers and seemed to be the most crucial to evaluate the chromaticity. In this study, the CQS of remote phosphor structures are compared in Figure 5. If the DL has the highest CRI, the TL reaches the highest CQS, which can be explained by the balance among the three colors red, yellow and green. The higher the CQS, the higher the color quality. Conversely, the CQS is found lower in DL structure. In general, TL structure is advantageous to the luminous flux, but hard to manage the color quality if there is no presence of the added red and green light components.

Based on Figure 5, it can be assured that if the goal of manufacturers is the color quality, they should select TL structure. Nevertheless, the rising question is that if the chromatic quality is better, is the luminous output affected? To figure out this question, the research team made a comparison of the emitted luminous flux between the DL and TL configurations. The part below presents the mathematical systems that were applied to determine results of blue light transmission and yellow light conversion in the TL remote structure. The results are studied to confirm the enhancement of LED efficiency achieved from this structure.

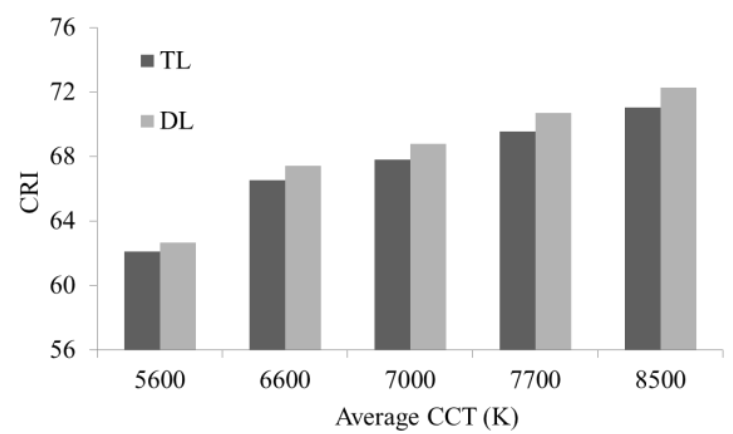

Figure 4. Color rendering indexes of phosphor configurations corresponding to ACCTs

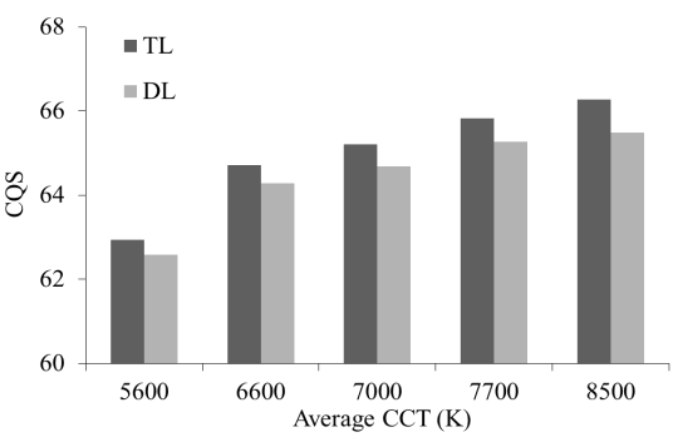

Figure 5. Color quality scale of phosphor configurations corresponding to ACCTs 
The emitted blue light and yellow light from light conversion for remote phosphor structure containing one phosphor layer with thickness described as $2 h$ are expressed as follows [24, 25]:

$$
\begin{aligned}
& P B_{2}=P B_{0} e^{-\alpha_{B_{2}} h} e^{-\alpha_{B_{2}} h}=P B_{0} e^{-2 \alpha_{B_{2}} h} \\
& P Y_{2}=\frac{1}{2} \frac{\beta_{2} P B_{0}}{\alpha_{B_{2}}-\alpha_{Y_{2}}}\left[e^{-\alpha_{Y_{2}} h}-e^{-\alpha_{B_{2}} h}\right] e^{-\alpha_{Y_{2}} h}+\frac{1}{2} \frac{\beta_{2} P B_{0}}{\alpha_{B_{2}}-\alpha_{Y_{2}}}\left[e^{-\alpha_{Y_{2}} h}-e^{-\alpha_{B_{2}} h}\right] \\
& \quad=\frac{1}{2} \frac{\beta_{2} P B_{0}}{\alpha_{B_{2}}-\alpha_{Y_{2}}}\left[e^{-2 \alpha_{Y_{1}} h}-e^{-2 \alpha_{B_{1}} h}\right]
\end{aligned}
$$

The emitted blue light and yellow light from light conversion for remote phosphor package containing two phosphor layers with the layer thickness of $h$ are measured with:

$$
\begin{aligned}
P B_{3}= & P B_{0} \cdot e^{-\alpha_{B_{2}} \frac{2 h}{3}} \cdot e^{-\alpha_{B_{2}} \frac{2 h}{3}} \cdot e^{-\alpha_{B_{2}} \frac{2 h}{3}}=P B_{0} \cdot e^{-2 \alpha_{B_{3}} h} \\
P Y^{\prime}{ }_{3}= & \frac{1}{2} \frac{\beta_{3} P B_{0}}{\alpha_{B_{3}}-\alpha_{Y_{3}}}\left[e^{-\alpha_{Y_{3}} \frac{2 h}{3}}-e^{-\alpha_{B_{3}} \frac{2 h}{3}}\right] e^{-\alpha_{Y_{3}} \frac{2 h}{3}}+\frac{1}{2} \frac{\beta_{3} P B_{0} e^{-\alpha_{B_{3}} \frac{2 h}{3}}}{\alpha_{B_{3}}-\alpha_{Y_{3}}} \\
= & \frac{1}{2} \frac{\beta_{3} P B_{0}}{\alpha_{B_{3}}-\alpha_{Y_{3}}}\left[e^{-\alpha_{Y_{3}} \frac{2 h}{3}}-e^{-2 \alpha_{B_{3}} \frac{2 h}{3}}\right] \\
P Y_{3}= & P Y^{\prime}{ }_{3} \cdot e^{-\alpha_{Y_{3}} \frac{2 h}{3}}+P B_{0} \cdot e^{-2 \alpha_{B_{3}} \frac{4 h}{3}} \frac{1}{2} \frac{\beta_{3}}{\alpha_{B_{3}}-\alpha_{Y_{3}}}\left[e^{-\alpha_{Y_{3}} \frac{2 h}{3}}-e^{-\alpha_{B_{3}} \frac{2 h}{3}}\right] \\
= & \frac{1}{2} \frac{\beta_{3} P B_{0}}{\alpha_{B_{3}}-\alpha_{Y_{3}}}\left[e^{-\alpha_{Y_{3}} \frac{4 h}{3}}-e^{-\alpha_{B_{3}} \frac{4 h}{3}}\right] e^{-\alpha_{Y_{3}} \frac{2 h}{3}}+\frac{1}{2} \frac{\beta_{3} P B_{0} e^{-\alpha_{B_{3}} \frac{4 h}{3}}}{\alpha_{B_{3}}-\alpha_{Y_{3}}} \\
= & \frac{1}{2} \frac{\beta_{3} P B_{0}}{\alpha_{B_{3}}-\alpha_{Y_{3}}}\left[e^{-\alpha_{Y_{3}} \frac{2 h}{3}}-e^{-\alpha_{B_{3}} h}-e^{-2 \alpha_{B_{3}} h}\right]
\end{aligned}
$$

The $h$ depicts the phosphor layer density. The subscript " 1 " and " 2 " note the single layer and double-layer remote phosphor configurations. $\beta$ is the conversion coefficient for blue light converting to yellow light. $\gamma$ is the reflection coefficient of the yellow light. The intensities of blue light $(P B)$ and yellow light $(P Y)$ are results obtained from light intensity of blue LED, indicated by $P B_{0} . \alpha_{B} ; \alpha_{Y}$ are indices describing the loss of transmitted energy in blue and yellow lights during their scattering process in their respective phosphor layer. Besides, in subscript " 4 ", $P Y_{3}{ }_{3}$ is the amount of yellow light that has passed two phosphor materials.

In TL remote structure pc-LEDs, there is a notable improvement in lighting efficiency when compared to a dual-layer one that can be seen from the following:

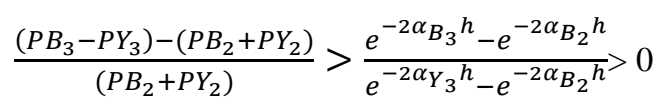

The Mie-theory is used in the analysis of scattering of phosphor particles. Moreover, through the following expression that applied the Mie theory, the scattering cross section $C_{s c a}$ for circular particles is obtained. In addition, the efficiency of light power transmission can be defined with Beer's law [25]:

$$
I=I_{0} \exp \left(-\mu_{e x t} L\right)
$$

The $I_{0}$ in this equation is the energy of incident light, $L$ represents the density of phosphor layer (mm) and $\mu_{\text {ext }}$ is the extinction coefficient, which are measured by: $\mu_{e x t}=N_{r}$. $C_{e x t}$, where $N_{r}$ is as the number density distribution of particles $\left(\mathrm{mm}^{-3}\right) . C_{\text {ext }}\left(\mathrm{mm}^{2}\right)$ is the extinction cross-section of phosphor particles.

In (6) proved three phosphor layers is more beneficial to the luminous flux than two layers. This is demonstrated in details with Figure 6. Specifically, TL structure shows higher lumen output than the DL does at all ACCTs. This erases suspicions about the benefits that TL brings to the luminous flux though it has the best color quality. In other words, TL structure can enhance both chromatic uniformity and lumen efficiency simultaneously. This finding can be explained by the higher emission intensity of TL in the wavelength range of $500 \mathrm{~nm}-600 \mathrm{~nm}$, compared to that of DL, due to a larger reduction in the YAG:Ce ${ }^{3+}$ 
phosphor concentration to keep the ACCT. At that time, the TL structure can reduce the internal back-scattering lights, leading to the blue light from LED chips can easily transmit through YAG:Ce ${ }^{3+}$ layer to other layers. In other words, TL structure helps the blue light power from LED chips to be converted effectively. Thus, compared to DL model, TL structure achieves higher emission spectrum intensity in the wavelength band of white light, resulting in better performance in its luminous flux. Hence, the TL structure can be chosen due to its superior in optical properties of WLEDs, including CQS and LE.

There are various methods to improve the color uniformity, which is an important element for color quality, such as scattering enhancement particles $\mathrm{SiO}_{2}, \mathrm{CaCO}_{3}$, etc., or conformal phosphor structure. When applying the green $\mathrm{Ca}_{2} \mathrm{La}_{2} \mathrm{BO}_{6.5}: \mathrm{Pb}^{2+}$ phosphor and red $\mathrm{Mg}_{8} \mathrm{Ge}_{2} \mathrm{O}_{11} \mathrm{~F}_{2}: \mathrm{Mn}^{4+}$ phosphor, the scattering properties and chromatic light components inside WLEDs are increased, leading to generating better white light. Additionally, utilizing the remote phosphor structure can promote the emitted luminous flux due to the reduction in the back-scattering of light to the LED chip. Although these two approaches can improve the color uniformity, the luminous flux seems to decrease. Therefore, the phosphor concentration needs to be adjusted to an appropriate amount to achieve the highest power transmission, which can be proven by the Lambert-Beer law in (7). In Figure 7 is the comparison of color deviation between DL and TL structures. As can be seen, the color deviation of TL structure is much smaller than that of DL structure, especially, at high ACCTs such as $8500 \mathrm{~K}$. The fact is that the smaller the deviation is, the higher the color uniformity becomes; and this statement can be demonstrated by the internal scattering of WLEDs: when more phosphor layers are applied, more scattering events occur. As a result, the chromatic uniformity of WLEDs increases. However, the rise in scattering event can lead to a decline in the luminous output. Nevertheless, this decrease is insignificant compared to the advantages given to WLEDs when the back-scattering is reduced. Therefore, the TL structure can attain either the best color uniformity or the highest luminous flux.

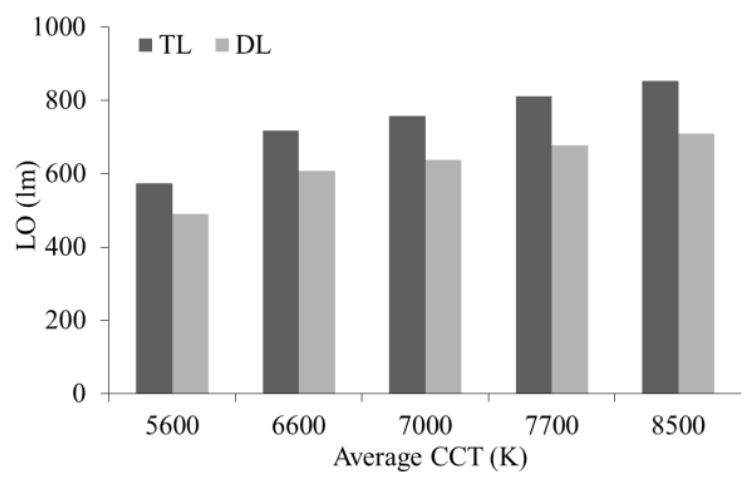

Figure 6. Luminous output (LO) of phosphor configurations corresponding to ACCTs

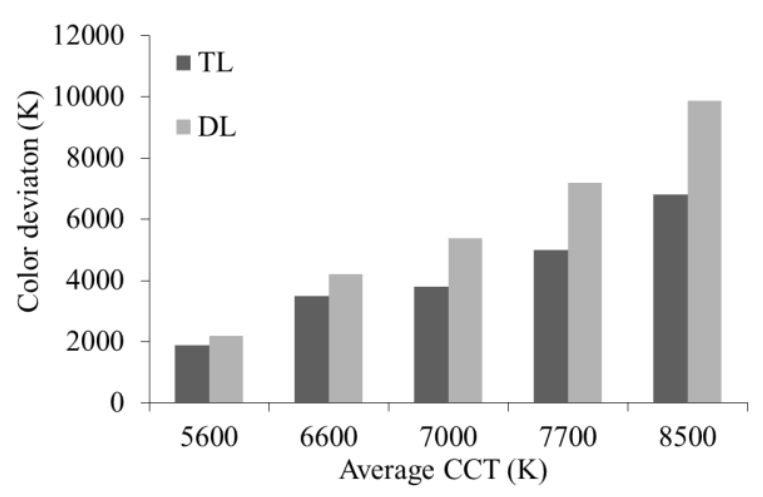

Figure 7. Correlated color temperature deviation (D-CCT) of remote phosphor configurations corresponding to ACCTs

\section{CONCLUSION}

This article compares the optical efficiency of the structures DL and TL at five ACCTs. The green $\mathrm{Ca}_{2} \mathrm{La}_{2} \mathrm{BO}_{6.5}: \mathrm{Pb}^{2+}$ and red $\mathrm{Mg}_{8} \mathrm{Ge}_{2} \mathrm{O}_{11} \mathrm{~F}_{2}: \mathrm{Mn}^{4+}$ phosphors are applied during the simulating process. Besides, the research results are verified by the Mie theory and the Lambert-Beer law. According to the results, adding the green $\mathrm{Ca}_{2} \mathrm{La}_{2} \mathrm{BO}_{6.5}: \mathrm{Pb}^{2+}$ phosphor layer increases the green light component to get the color uniformity, and luminous output enhanced. Thus, the TL structure results in better luminous flux and chromatic uniformity than the DL one. Moreover, color rendering index and color quality scale, two most important color quality indicators are improved with the presence of red $\mathrm{Mg}_{8} \mathrm{Ge}_{2} \mathrm{O}_{11} \mathrm{~F}_{2}: \mathrm{Mn}^{4+}$ phosphor in the configuration. The result shows that the TL structure has lower CRI but better and CQS than the DL. It can be easily seen that the chromaticity depends on the balance among the three primary colors yellow, red, and green. Therefore, the TL turns out to be the best selection in controlling these three colors. Besides, the reduction in back-scattering of TL structure causes its luminous flux to increase noticeably. The proof is that TL presents the highest value of luminous efficiency. In other words, TL structure can increase the luminous efficiency and color quality of WLEDs at the same time, which could not be accomplished by using DL structure. Based on the results of this study, manufacturers can easily choose the most appropriate structure to enhance the quality of WLEDs. 


\section{REFERENCES}

[1] S. Pan, et al., "Image restoration and color fusion of digital microscopes," Appl. Opt., vol. 58, pp. 2183-2189, 2019.

[2] O. Kunieda and K. Matsushima, "High-quality full-parallax full-color three-dimensional image reconstructed by stacking large-scale computer-generated volume holograms," Applied Optics, vol. 58, pp. G104-G111, 2019.

[3] Q. Zaman, et al., "Two-color surface plasmon resonance nanosizer for gold nanoparticles," Optics Express, vol. 27, pp. 3200-3216, 2019.

[4] D. Durmus and W. Davis, "Blur perception and visual clarity in light projection systems," Optics Express, vol. 27, pp. A216-A223, 2019.

[5] R. Hirayama, et al., "Projection of multiple directional images on a volume structure with refractive surfaces," Optics Express, vol. 27, pp. 27637-27648, 2019.

[6] T. Z. Wu, et al., "Analyses of multi-color plant-growth light sources in achieving maximum photosynthesis efficiencies with enhanced color qualities," Optics Express, vol. 26, pp. 4135-4147, 2018.

[7] Y. L.Piao, et al., "Chromatic-dispersion-corrected full-color holographic display using directional-view image scaling method," Appl. Opt., vol. 58, pp. A120-A127, 2019.

[8] P. Zhu, et al., "Design of circadian white light-emitting diodes with tunable color temperature and nearly perfect color rendition," OSA Continuum, vol. 2, pp. 2413-2427, 2019.

[9] D. Lin, et al., "Silicon solar cells efficiency enhanced in NIR band by coating plasmonics ITO- and UC phosphorsparticles layers on back-side surface using spin-on film deposition," CLEO: Applications and Technology Optical Society of America, 2019.

[10] T. Li, et al., "Efficient X-ray excited short-wavelength infrared phosphor," Optics Express, vol. 27, pp. 13240-13251, 2019.

[11] Q. T. Fouliard, et al., "Modeling luminescence behavior for phosphor thermometry applied to doped thermal barrier coating configurations," Applied Optics, vol. 58, 2019.

[12] Z. J. Zhang, et al., "Tunable photoluminescence in Ba1-xSrxSi3O4N2: Eu2+/ Ce3+, Li+ solid solution phosphors induced by linear structural evolution," Optical Materials Express, vol. 9, pp. 1922-1932, 2019.

[13] A. K. Dubey, et al., "Laser-line-driven phosphor-converted extended white light source with uniform illumination," Applied Optics, vol. 58, pp. 2402-2407, 2019.

[14] G. Zhang, et al., "Spectral optimization of color temperature tunable white LEDs with red LEDs instead of phosphor for an excellent IES color fidelity index," OSA Continuum, vol. 2, pp. 1056-1064, 2019.

[15] B. Wang, et al., "Eu3+ doped high-brightness fluorophosphate laser-driven glass phosphors," Optical Materials Express, vol. 9, pp. 1749-1762, 2019.

[16] F. Steudel, et al., "Pixelated phosphors for high-resolution and high-contrast white light sources: erratum," Optics Express, vol. 27, pp. 9097-9098, 2019.

[17] B. Fond, et al., "Investigation of the tin-doped phosphor (Sr,Mg)3(PO4)2:Sn2+ for fluid temperature measurements," Optical Materials Express, vol. 9, pp. 802-818, 2019.

[18] H. Yuce, et al., "Phosphor-based white LED by various glassy particles: control over luminous efficiency," Optics Letters, vol. 44, pp. 479-482, 2019.

[19] W. Wang, et al., "Red photoluminescent Eu3+-doped Y2O3 nanospheres for LED-phosphor applications: Synthesis and characterization," Optics Express, vol. 26, pp. 34820-34829, 2018.

[20] T. Wei, et al., "Single Pr3+-activated high-color-stability fluoride white-light phosphor for white-light-emitting diodes," Optical Materials Express, vol. 9, pp. 223-233, 2019.

[21] A. Dwivedi, et al., "Monochromatic NIR UC emission in Tm3+/Yb3+co-doped GdVO4 phosphor: the effect of the Bi3+ ion concentration and pump power of a diode laser," Optics Letters, vol. 43, pp. 5785-5788, 2018.

[22] J. S. Li, et al., "High efficiency solid-liquid hybrid-state quantum dot light-emitting diodes," Photonics Research, vol. 6, pp. 1107-1115, 2018.

[23] A. Zhang, et al., "Tunable white light emission of a large area film-forming macromolecular complex with a high color rendering index," Optical Materials Express, vol. 8, pp. 3635-3652, 2018.

[24] B. Li, et al., "High-efficiency cubic-phased blue-emitting Ba3Lu2B6O15:Ce3+ phosphors for ultraviolet-excited white-light-emitting diodes," Optics Letters, vol. 43, pp. 5138-5141, 2018.

[25] X. Wang, et al., "Broadband multicolor upconversion from $\mathrm{Yb} 3+-\mathrm{Mn} 2+$ codoped fluorosilicate glasses and transparent glass ceramics," Optics Letters, vol. 43, pp. 5013-5016, 2018. 